\title{
BMJ Open Prehospital triage of patients with acute cardiac complaints: study protocol of HART-c, a multicentre prospective study
}

\author{
Enrico de Koning (D) , ${ }^{1}$ Tom E Biersteker, ${ }^{1}$ Saskia Beeres, ${ }^{1}$ Jan Bosch, ${ }^{2}$ \\ Barbra E Backus, ${ }^{3}$ Charles JHJ Kirchhof, ${ }^{4}$ Reza Alizadeh Dehnavi, ${ }^{5}$ \\ Helen AM Silvius, ${ }^{6}$ Martin Schalij, ${ }^{1}$ Mark J Boogers ${ }^{1}$
}

To cite: de Koning $E$, Biersteker TE, Beeres S, et al. Prehospital triage of patients with acute cardiac complaints: study protocol of HART-c, a multicentre prospective study. BMJ Open 2021;11:e041553. doi:10.1136/ bmjopen-2020-041553

- Prepublication history and additional material for this paper are available online. To view these files, please visit the journal online (http://dx.doi. org/10.1136/bmjopen-2020041553).

Received 17 June 2020 Revised 20 January 2021 Accepted 28 January 2021

Check for updates

(C) Author(s) (or their employer(s)) 2021. Re-use permitted under CC BY-NC. No commercial re-use. See rights and permissions. Published by BMJ.

For numbered affiliations see end of article.

Correspondence to Dr Mark J Boogers; j.m.j.boogers@lumc.nl

\section{ABSTRACT}

Introduction Emergency department (ED) overcrowding is a major healthcare problem associated with worse patient outcomes and increased costs. Attempts to reduce ED overcrowding of patients with cardiac complaints have so far focused on in-hospital triage and rapid risk stratification of patients with chest pain at the ED. The Hollands-Midden Acute Regional Triage-Cardiology (HART-C) study aimed to assess the amount of patients left at home in usual ambulance care as compared with the new prehospital triage method. This method combines paramedic assessment and expert cardiologist consultation using live monitoring, hospital data and realtime admission capacity.

Methods and analysis Patients visited by the emergency medical services (EMS) for cardiac complaints are included. EMS consultation consists of medical history, physical examination and vital signs, and ECG measurements. All data are transferred to a newly developed platform for the triage cardiologist. Prehospital data, in-hospital medical records and real-time admission capacity are evaluated. Then a shared decision is made whether admission is necessary and, if so, which hospital is most appropriate. To evaluate safety, all patients left at home and their general practitioners (GPs) are contacted for 30-day adverse events.

Ethics and dissemination The study is approved by the LUMC's Medical Ethics Committee. Patients are asked for consent for contacting their GPs. The main results of this trial will be disseminated in one paper.

Discussion The HART-c study evaluates the efficacy and feasibility of a prehospital triage method that combines prehospital patient assessment and direct consultation of a cardiologist who has access to live-monitored data, hospital data and real-time hospital admission capacity. We expect this triage method to substantially reduce unnecessary ED visits.

\section{INTRODUCTION}

Emergency department (ED) overcrowding is a worldwide healthcare problem associated with worse patient outcomes and increased costs. ${ }^{2}$ Cardiac complaints are one of the most common reasons for patients
Strengths and limitations of this study

- A novel prehospital triage method is presented, which aims to safely decrease unnecessary emergency department admissions, using telemedicine for prehospital decision making.

- All participants, including patient representatives, were involved in the design of the study.

- This study is a real-life reflection of interdisciplinary daily clinical practice; the retrospective cohort will reflect the control group in the best way possible.

- The Hollands-Midden Acute Regional TriageCardiology study is a non-randomised controlled trial and therefore has risk of bias as decisions from paramedics and cardiologists can be influenced by the study.

to visit the $\mathrm{ED}$, with chest pain as the most frequent complaint. ${ }^{3}$ In Europe and the USA, 15-20 million patients with chest pain are seen at the ED every year. ${ }^{4}$ The majority will be sent home after ruling out acute cardiovascular disease: previous studies have shown that up to $80 \%$ of patients with chest pain do not have an acute coronary syndrome. ${ }^{5-8}$ However, these patients contribute to overcrowding of EDs and these ED visits substantially increase healthcare costs.

Attempts to reduce ED overcrowding by patients with cardiac complaints have so far particularly focused on rapid risk stratification after presentation at the ED. For example, the HEART (History, ECG, Age, Risk Factors, Troponin) score stratifies patients as at low, intermediate or high risk of major adverse cardiac events (MACEs) based on history, the ECG, age, risk factors and troponin levels. ${ }^{9}$ However, as it takes 1-2 hours for the latter to be available, patients still spend a long time at the ED, after which the majority can be discharged home. 
Accordingly, interest has shifted from in-hospital to prehospital triage. Preventing patients with cardiac complaints and a very low risk of adverse cardiac events from visiting the ED will substantially help to reduce ED overcrowding. Efforts to prevent ED visits especially involve interventions focused on patients with chest pain, such as risk score calculation by the ambulance paramedics (eg, with the HEART score ${ }^{10}$ and History and Electrocardiogram-Only Manchester Acute Coronary Syndrome (HE-MACS) ${ }^{11}$ or prehospital point of care testing for troponin. ${ }^{12}$ In order to improve prehospital triage for patients with cardiac complaints in the entire chain of acute cardiac care, we developed a comprehensive triage method entitled Hollands-Midden Acute Regional Triage-Cardiology (HART-c).

Innovative in this approach is the combination of prehospital patient assessment by the ambulance paramedic and expert consultation of a cardiologist who has access to live-monitored data from the ambulance, in-hospital data and real-time hospital admission capacity in a newly developed triage application. By drafting this triage method, we specifically aimed to safely reduce unnecessary ED visits of patients with all types of cardiac complaints. In addition, we intend to provide patienttailored care through prehospital assessment of patientspecific needs and circumstances. The HART-c study was designed to evaluate whether the implementation of the HART-c triage method results in a reduction of unnecessary ED visits.

\section{METHODS AND ANALYSIS}

\section{Study design and patient population}

The HART-c study is a multicentre prospective study with a historical control group. The intervention group comprises adult patients visited by the regional emergency medical services (EMS) because of cardiac complaints between 1 September 2019 and 31 August 2020 in whom prehospital triage is performed according to the HART-c triage method. The historical control groups consist of adult patients visited by the regional EMS because of cardiac complaints between 1 September 2018 and 31 August 2019 (1 year before the start of the HART-c triage method). Of note, in both groups, EMS consultation could have been requested directly by the patient, through bystanders or by the patients' general practitioner (GP), who refers patients through EMS. Patients in need of urgent cardiac care, patients with complaints not suspected of cardiac origin as assessed by the ambulance paramedic and patients unable or not willing to provide informed consent were excluded from triage according to the HART-c method. Box 1 displays the detailed inclusion and exclusion criteria.

The HART-c study is coordinated by the Leiden University Medical Center (LUMC) and conducted in the entire EMS region Hollands-Midden, which consists of over 600000 inhabitants. The hospitals located in this region that participated included the LUMC, the Groene Hart
Box 1 Inclusion and exclusion criteria

\section{Inclusion criteria}

Patients visited by emergency medical services for cardiac complaints

\section{Age over 18 years}

\section{Exclusion criteria}

Patients in need fof urgent cardiac care because of

- ST-elevation myocardial infarction.

- Haemodynamic instability.

- (Out of hospital) cardiac arrest.

- Suspected pulmonary embolism.

- Suspected acute aortic syndrome (thoracic or abdominal).

Patients with symptoms not suspected of cardiac origin

Unable or unwilling to provide informed consent

Hospital and the Alrijne hospital. The study is performed in close collaboration with the regional EMS (Regional Ambulance Service Hollands-Midden), which employs 240 paramedics who are trained in prehospital cardiac care and 31 ambulance vehicles equipped with real-time monitoring. In addition, the study was performed and developed with the help of over regional 300 GPs.

\section{Intervention group: prehospital triage using HART-c method} The intervention group consists of patients visited by the EMS because of symptoms suspected to be of cardiac origin, such as chest pain, shortness of breath, palpitations or implanted cardiac device problems. In line with the National Protocol for Emergency Medical Care, patients at first receive standard medical care consisting of a medical history, physical examination with vital sign monitoring (blood pressure, heart rate and pulse oximetry) and a 12-lead ECG. ${ }^{13}$ In patients with chest pain, the prehospital modified HEART score (the HEART ${ }^{14}$ score without troponin) is calculated. All acquired data are noted on a handheld device and stored on AmbuSuite, an external secure database. Afterwards, the ambulance paramedic directly contacts the on-call triage cardiologist. The right panel in figure 1 illustrates the entire routing of patients in the intervention group. In total, 43 cardiologists from all three regional hospitals are scheduled so one cardiologist is on duty for the entire region. GPs can refer patients through EMS consultation; however, in the intervention period, cardiologist consultation is possible. When GPs are in doubt of referral, they can request cardiologist consultation through EMS with the HART-c method. The triage cardiologist evaluates the prehospital data, including medical history, real-time vital parameters and 12-lead ECG, and combines them with (if present) previous medical records and the actual hospital admission capacity of the regional hospitals. Also, we developed decision aids for chest pain, dyspnoea and arrhythmia as guidance for triage cardiologists. These decision aids can help the triage cardiologist in decision making and are 
A

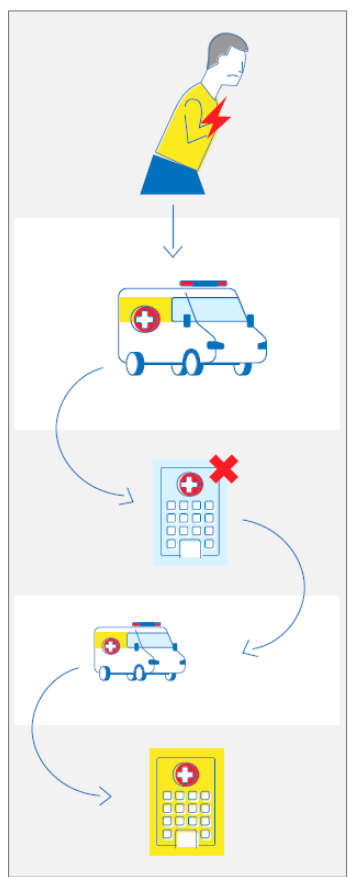

B

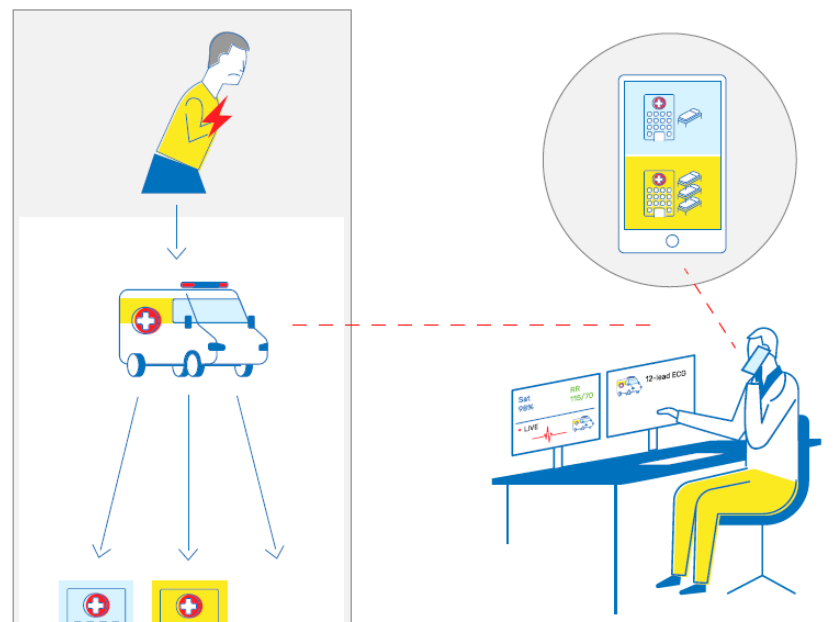

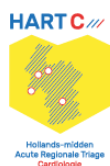

Figure 1 Method of triage. (A) Patient routing without prehospital selection where patients are referred to the nearest emergency department or left at home. if hospital admission capacity is insufficient, patients are transferred to another hospital. (B) Patient routing with prehospital selection using prehospital and in-hospital data where a cardiologist has insight in live vital parameters and regional hospital capacity.

added as online supplemental figure 1 for chest pain, online supplemental figure 2 for dyspnoea and online supplemental figure 3 for arrhythmia to this article. Based on these comprehensive data, the triage cardiologist and ambulance paramedic decide, as a shared decision with the patient, whether transfer to an ED is necessary and, if so, which hospital and which department is most suitable. The triage decision is sent immediately to the responsible

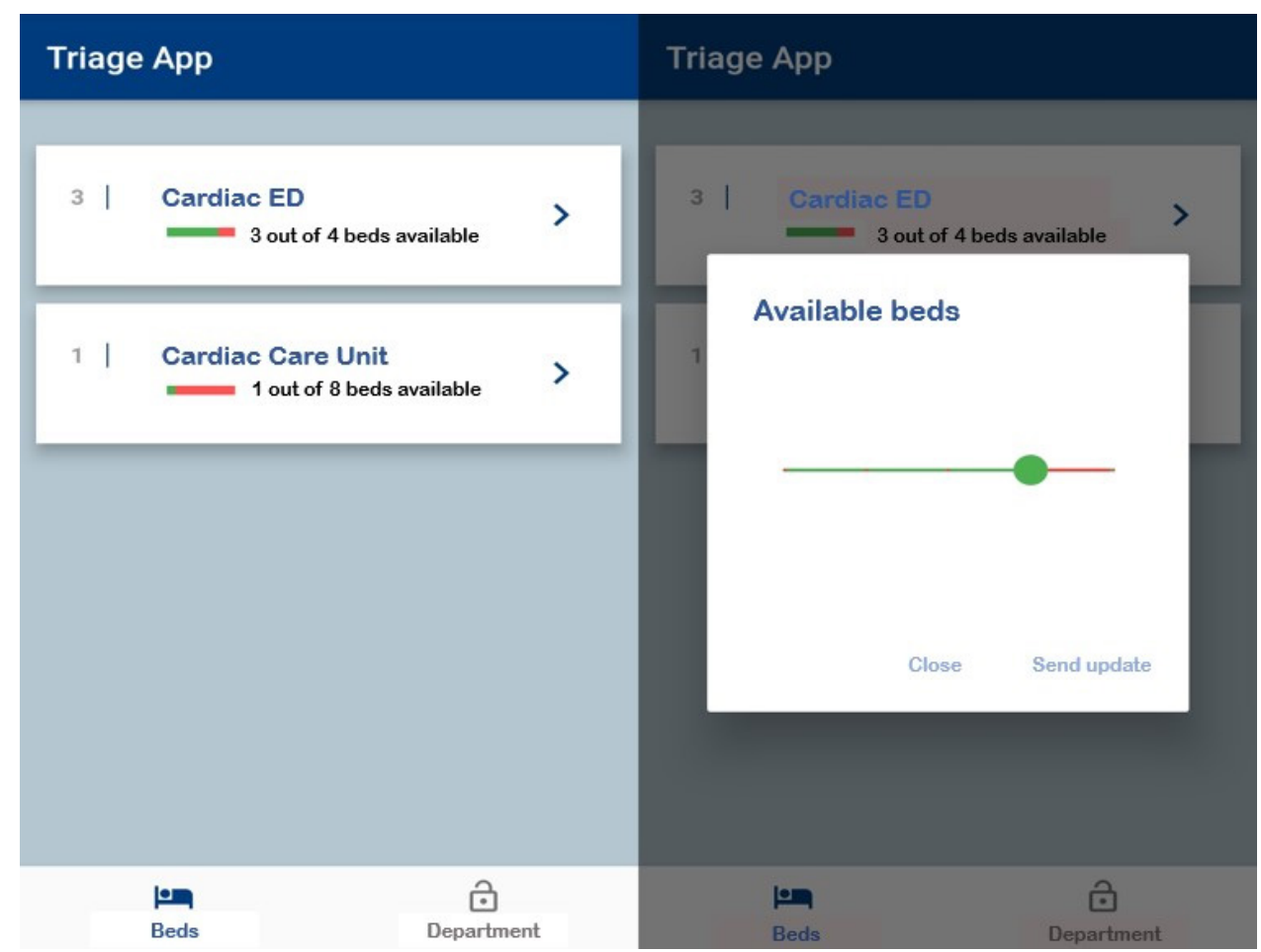

Figure 2 Mobile phone triage application: left panel shows an overview of a hospital-specific capacity; right panel shows the ability to update capacity. 


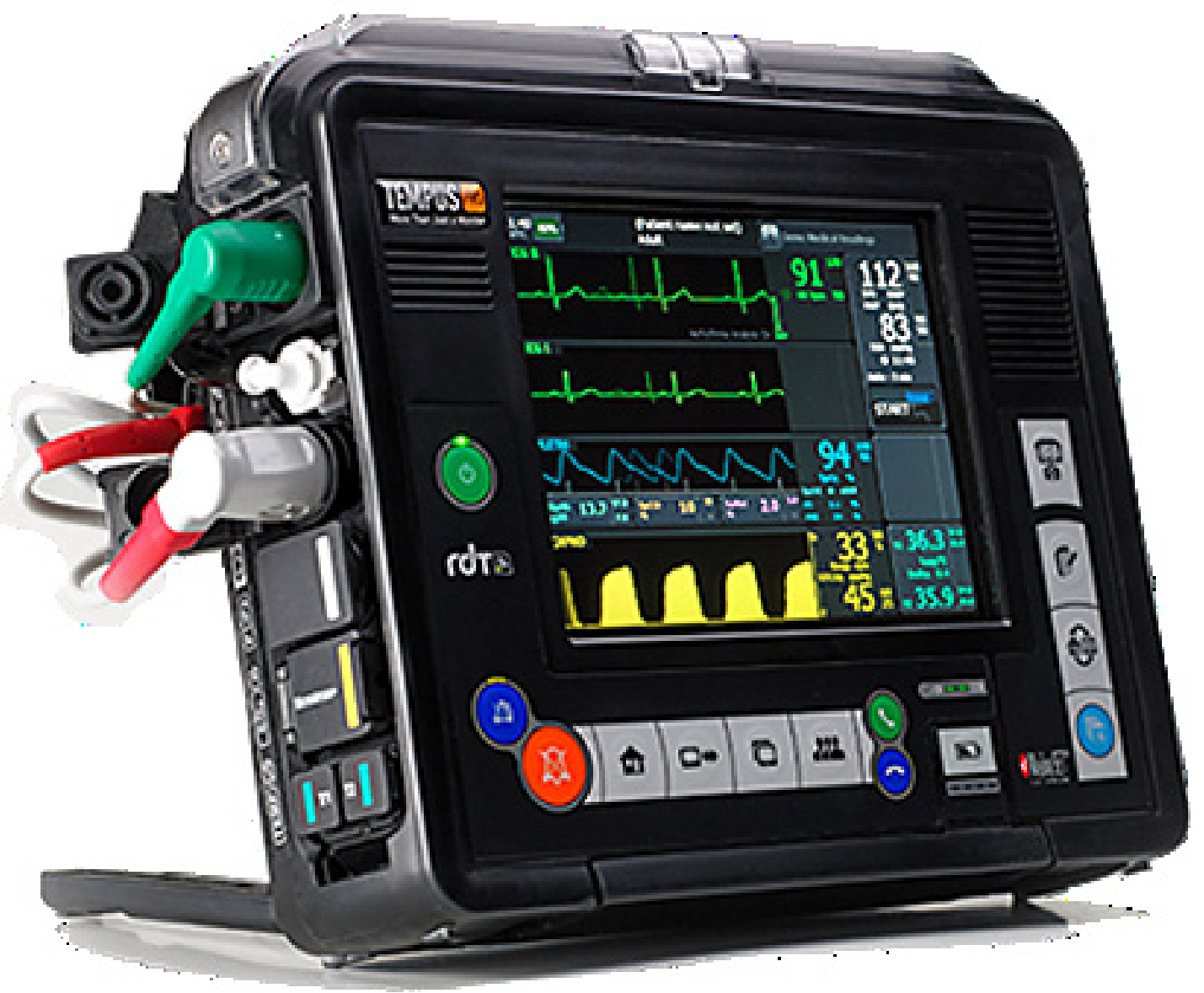

Figure 3 Image of Tempus pro monitor.

ED nursing staff and the capacity of this hospital is updated automatically (figure 2). On arrival at the ED, cardiac assessment is based on in-house clinical decision rules as guidelines prescribe, 12-lead ECG and laboratory findings. ${ }^{9}$

Intervention group: Tempus Pro Monitor, IntelliSpace Corsium, triage platform and data handling

All ambulances are equipped with a Tempus Pro Monitor ${ }^{15}$ (Philips, The Netherlands), which allows recording of a 12-lead ECG and real-time monitoring of the following vital patient parameters: heart rate, blood pressure and pulse oximetry. The monitor can show trends in measurements and stream data for up to 10 hours. All data are encrypted and shared with the on-call triage cardiologist through secure channels. The Tempus Pro Monitor is shown in figure 3 .

Using a secure log-in, the on-call triage cardiologist logs in to IntelliSpace Corsium (Philips, the Netherlands) and connects digitally with a patient-specific Tempus Pro Monitor. All aforementioned measurements are streamed live. Once the live streaming ends, no patient-specific data are stored on the platform. This system of dat transfer is Food and Drug Administration (FDA) approved. ${ }^{1617}$

A novel triage platform was developed showing realtime admission capacity of the regional hospitals. The nursing staff in these hospitals continuously updates their admission capacity. Linking these capacity data to a local electronic patient dossier (EPD; Vision, Leiden, The Netherlands), the on-call triage cardiologist obtains insight into the actual bed occupancy of each hospital. After consultation, the on-call triage cardiologist notes his decision and a message is automatically sent to the nursing staff of the chosen hospital, thereby updating their admission capacity immediately.

Patient data are sent securely from Tempus Monitor to IntelliSpace Corsium. No data are stored on IntelliSpace Corsium. No patient data are transmitted to the mobile phone application. Patient data are transferred from AmbuSuite to our EPD. All patient data and decisions are stored on the EPD of the coordinating hospital and are only accessible for triage cardiologists.

\section{Historical control group: standard care in prehospital setting} The historical control group consists of patients visited by the EMS because of potential cardiac complaints in the year before the onset of the HART-c triage method. On arrival by the paramedic, standard medical care consists of medical history, physical examination with vital sign monitoring (blood pressure, heart rate and pulse oximetry) and a 12-lead ECG. All acquired data are noted on a handheld device and stored on AmbuSuite (Topicus, 
the Netherlands), which is an external secure database. Thereafter, the ambulance paramedic decides, based on predefined national protocols and decision rules for diagnosis, whether transfer to an ED is deemed necessary. ${ }^{12}$ Paramedics are able to identify low-risk patients for all medical specialties and decide whether admission or ED presentation is necessary on every consultation. So, even in the historical cohort group, only patients with cardiac complaints deemed severe enough for presentation are presented to the ED. Of note, at the time of referral, the paramedic has no insight in the previous medical records and the actual hospital admission capacity. The Netherlands has a unique system where, in the historical cohort in our region, approximately $5 \%$ of patients with cardiac complaints are not referred to a hospital after paramedic assessment; instead these patients are directly referred to their GP or treated at home. However, given the number of unnecessary ED visits, there is still a large cohort of low-risk patients in whom ED presentation could be prevented. After paramedic assessment and hospital transport, cardiac assessment on the ED is based on in-house clinical decision rules as guidelines prescribe, 12-lead ECG and laboratory findings. ${ }^{9}$ If evaluation at the ED indicates that hospitalisation is mandatory, the patient is admitted in the concerned hospital. However, when admission capacity is insufficient or immediate intervention is not available in the concerned hospital, ambulance transfer to another hospital is mandatory. The routing of patients in the historical control group is illustrated in the left panel of figure 1 .

\section{Objective and outcome measures}

The HART-c study is designed to evaluate the efficacy and feasibility of a novel comprehensive prehospital triage method which aims to safely reduce unnecessary ED visits in patients with cardiac complaints. The primary outcome is the percentage of patients in whom an ED visit can be prevented after EMS consultation. The following secondary endpoints will be evaluated:

- Number of ambulance transfers to an ED because of cardiac complaints.

- Number of interhospital transfers in patients with cardiac complaints.

- Patient, triage cardiologist and GP satisfaction with the HART-c triage method on a $0-10$ scale.

- Time from EMS consultation to arrival at the hospital in the both study groups.

- Safety of the HART-c prehospital triage method. This will be evaluated in patients in the intervention group who are not transferred to an ED after cardiologist consultation. Safety will be assessed by the occurrence of adverse events up to 30 days of follow-up. Box 2 displays the prespecified major and nonmajor adverse events. To evaluate safety, a dedicated researcher will contact these patients and their GP and evaluate on a case-by-case basis. If a major adverse event is deemed directly attributable to the triage method, the protocol will be adjusted or the study will
Box 2 Adverse events (30 days after emergency medical services (EMS) contact)

Major adverse events
Death.
Acute coronary syndrome.
Other adverse events
Renewed EMS or emergency department (ED) visit for cardiac
complaint.
Pulmonary embolism.
ED visit or hospitalisation for acute decompensated heart failure.
Ventricular tachycardia or fibrillation.
Cerebrovascular accident or transient ischaemic attack.

be terminated prematurely. The study will be deemed safe if the percentage of major adverse events is $1 \%$ or lower.

- Feasibility of the HART-c prehospital triage method. This will be evaluated in the intervention group and defined as the absence of technical problems for the ambulance paramedic and the triage cardiologist. This means access to the live-monitored data from the ambulance, hospital data and real-time hospital admission capacity are all available. In order to swiftly manage potential technical problems, the HART-c triage method will start during working hours. If interim analysis reveals that the method is feasible, the time frame in which HART-c triage is available could be extended.

\section{Statistical analysis}

The prevention of an ED visit after EMS consultation will be analysed using a logistic regression analysis. Ambulance transfers and interhospital transfers in the intervention versus the control group will also be evaluated using logistic regression. Baseline characteristics will be reported as mean and SD or median and IQR and compared between historical cohort and intervention. This study will be underpowered to detect differences in mortality and MACEs. Accordingly, these events will only be reported and no further statistics on mortality and MACE will be done. The data will be analysed using IBM SPSS Statistics V.25. A p value lower than 0.05 will be considered statistically significant.

\section{Patient and public involvement}

Patients were involved in the design of the study. During the design stage, representatives from the 'Harteraad', a cardiovascular patient council, were asked for input in study design, choice of outcome measures and methods of recruitment. Also, a dedicated website (www.hartc.nl) was created to inform the public and to answer questions from professionals and patients, before and during the study.

\section{Ethics and dissemination}

The study is approved by the LUMC's Medical Ethics Committee (P18.213). Patients are requested to provide 
oral informed consent for contacting their GP at 30 days of follow-up. Oral informed consent is requested for cardiologist consultation and study participation by paramedics, which is then noted in AmbuSuite. The need for written informed consent was waived by the medical ethics committee. The devices used in this study are FDA and/or CE (European Conformity) approved. No manufacturer has a role in study design, data collection, statistical analysis or writing of the manuscript. No financial support is received for this study from any manufacturer. The main results of this trial will be disseminated in one paper.

\section{DISCUSSION}

Overcrowding of EDs is a major challenge in healthcare. The HART-c study is a multicentre prospective study that primarily aims to safely reduce unnecessary ED visits of patients with all types of cardiac complaints. By selecting the hospital best suited for every patient, this method will contribute to more patient-tailored healthcare and lead to improved use of all available healthcare resources in the region.

Recently, interest has shifted from in-hospital to prehospital triage. Prehospital cardiologist consultation has been the standard procedure for some time in many hospitals in the Netherlands for quick catheterisation lab activation when paramedics suspect patients with chest pain of ST-segment elevation myocardial infarction (STEMI). ${ }^{18}$ For all other cardiac complaints, no pre-hospital triage procedure is in place for emergency evaluations. However, there have been some studies assessing the possibility of prehospital triage and prehospital selection of low-risk patients with cardiac complaints.

The HE-MACS decision aid was developed for prehospital triage using history, physical examination and ECG. It was derived in 796 patients and validated in cohorts of 474 and 659 patients. Of all validated patients, $9.4 \%$ were identified as 'very low risk', in which ACS could be 'ruled out' with a sensitivity of $99.5 \%$. It' impact, however, was not prospectively evaluated in this study.

The FAMOUS investigators aim to assess the effects of introducing a pre-hospital triage system that stratifies patients with chest pain without ST segment elevation into (1) patients at high risk of NSTEMI (non-ST-segment elevation myocardial infarction) requiring direct transfer to a PCI (percutaneous coronary intervention) hospital, (2) patients at intermediate risk of MACEs who could be evaluated at the nearest non-PCI hospital and (3) patients at low risk of MACEs who could have further evaluation at home or in a primary care setting. ${ }^{19}$ The study was divided in three phases. In the first phase, a venous blood sample was drawn in the ambulance for measurement of the prehospital troponin $\mathrm{T}$ levels, in order to establish a prehospital HEART score and to evaluate the possibility of triage at the patient's home. Of the 1127 patients with chest pain, $36 \%$ had a low modified HEART score and none of them developed a major adverse event. ${ }^{20}$ After this first phase proving feasibility, further studies have been done in the prehospital setting by the FAMOUS TRIAGE (fast assessment and management of chest pain without ST-elevation in the pre-hospital gateway) study group. Phase II, a prospective observational study including 700 patients with suspected NSTEMI, showed nicely that prehospital risk stratification by ambulance paramedics using the HEART score was accurate in differentiating in low and intermediate to high risk. ${ }^{21}$ Recently, the design of phase III has been published, where the FAMOUS study investigators aim to determine if use of the HEART score, including point-of-care troponin measurement, is non-inferior to routine management. In this phase, referral decisions are based on prehospital acquired risk stratification. ${ }^{22}$

Another study investigating the added value of pointof-care troponin in the prehospital setting is the ARTICA

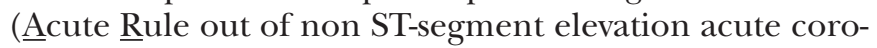
nary syndrome in the (pre)hospital setting by HEART score assessment and a single poInt of CAre troponin) ${ }^{12}$ trial. This randomised trial will include patients suspected of non-ST elevation acute coronary syndrome in whom the modified HEAR score (the HEART score without troponin) is calculated by the ambulance paramedic. If the HEAR score is less than or equal to 3, patients will be 1:1 randomised for (1) presentation at the ED or (2) point-of-care troponin $\mathrm{T}$ measurement and transfer of care to the GP in case of a low troponin $\mathrm{T}$ value. The primary objective of the ARTICA trial will be healthcare costs at 30 days. The trial is currently ongoing and aims to include 866 patients in 12 months.

The similarity of the currently described HART-c study and the HE-MACS, FAMOUS and ARTICA studies is that all three assess whether patients with chest pain who are at low risk of major adverse events can be identified before presenting to the ED. However, the HART-c study has some added benefit as opposed to earlier known studies. First, the HART-c study does not only identify patients at low risk of events but also aims to effectively prevent low-risk patients from actually visiting the ED, as well as further phases from FAMOUS and ARTICA did, by combining prehospital risk stratification by the paramedic and real-time cardiologist consultation with insight in live vital parameters and ECG. Second, while these studies study only focus on patients with chest pain, the HART-c study extends this to all patients with cardiac complaints and could therefore be of benefit for a substantially larger cohort of patients. Furthermore, the HART-c triage method is unique as it combines prehospital patient assessment by the ambulance paramedic and direct consultation of an expert triage cardiologist who has access to live-monitored data from the ambulance for all patients with cardiac complaints, as opposed to only STEMI patients. Besides these novelties, the HART-c study incorporates hospital data, as well as real-time hospital admission capacity, to decide which regional hospital is best suited for every patient. In the future, it would be helpful to have prehospital information integrated in all 
hospitals EPD. At this moment, however, that is not the case.

If the results of current study show that the HART-c triage method is effective in safely reducing unnecessary ED visits of patients with all types of cardiac complaints, the next step will be to evaluate cost-effectiveness. When cost-effectiveness can be demonstrated, we feel that the HART-c triage method can be expanded to other EMS regions. Furthermore, last but not least, it may potentially also be useful for other medical specialists aiming to optimise prehospital triage of patients without cardiac complaints. Eventual improvements in prehospital triage, such as prehospital high-sensitive troponin sampling with a point-of-care test or newly developed and proven risk scores, could always be implemented in this triage protocol.

To conclude, the HART-c study is a multicentre prospective study evaluating the efficacy and feasibility of a novel comprehensive prehospital triage method that combines prehospital patient assessment by the ambulance paramedic and direct consultation of a cardiologist who has access to live-monitored data from the ambulance, hospital data, as well as real-time hospital admission capacity. If the HART-c study will succeed to safely reduce unnecessary ED visits of patients with all types of cardiac complaints, it may help to decrease ED overcrowding and ultimately reduce healthcare expenditures.

\section{Author affiliations \\ ${ }^{1}$ Cardiology, Leiden University Medical Center, Leiden, The Netherlands \\ ${ }^{2}$ Research and Development, Regional Ambulance Service Hollands-Midden (RAVHM), Leiden, The Netherlands \\ ${ }^{3}$ Emergency Medicine, Leiden University Medical Center, Leiden, The Netherlands \\ ${ }^{4}$ Cardiology, Alrijne Hospital, Leiderdorp, The Netherlands \\ ${ }^{5}$ Cardiology, Groene Hart Hospital, Gouda, The Netherlands \\ ${ }^{6}$ Public Health and General Practice, Leiden University Medical Center, Leiden, The Netherlands}

Contributors EdK, TEB, JB, CJHJK, RAD, HAMS, MS and MJB made a substantial contribution to the concept and design of the work. EdK and MJB are in charge of acquisition of data, analysis and interpretation of the data. EdK, TEB, SB and MJB contributed to the drafting of the article. EdK, SB, JB, BEB, CJHJK, RAD, MS and $M J B$ all revised the manuscript critically for important intellectual content.

Funding The Department of Cardiology of the Leiden University Medical Center receives unrestricted research and educational grants from Boston Scientific, Medtronic and Biotronik outside the submitted work.

Competing interests EdK, TEB, SB, JB, CJHJK, RAD, HAMS, MS and MJB report no conflict of interest. BEB is the creator of the HEART score.

Patient consent for publication Not required.

Provenance and peer review Not commissioned; externally peer reviewed.

Supplemental material This content has been supplied by the author(s). It has not been vetted by BMJ Publishing Group Limited (BMJ) and may not have been peer-reviewed. Any opinions or recommendations discussed are solely those of the author(s) and are not endorsed by BMJ. BMJ disclaims all liability and responsibility arising from any reliance placed on the content. Where the content includes any translated material, BMJ does not warrant the accuracy and reliability of the translations (including but not limited to local regulations, clinical guidelines, terminology, drug names and drug dosages), and is not responsible for any error and/or omissions arising from translation and adaptation or otherwise.

Open access This is an open access article distributed in accordance with the Creative Commons Attribution Non Commercial (CC BY-NC 4.0) license, which permits others to distribute, remix, adapt, build upon this work non-commercially, and license their derivative works on different terms, provided the original work is properly cited, appropriate credit is given, any changes made indicated, and the use is non-commercial. See: http://creativecommons.org/licenses/by-nc/4.0/.

ORCID iD

Enrico de Koning http://orcid.org/0000-0002-9343-0717

\section{REFERENCES}

1 American College of Emergency Physicians Policy statements. Crowding. Ann Emerg Med 2006;47:585.

2 Boyle A, Beniuk K, Higginson I, et al. Emergency department crowding: time for interventions and policy evaluations. Emerg Med Int 2012;2012:1-8.

3 Bhuiya FA, Pitts SR, McCaig LF. Emergency department visits for chest pain and abdominal pain: United States, 1999-2008. NCHS Data Brief 2010;43:1-8.

4 Nawa EW, Nisk RW, Xu J. National Hospital ambulatory medical care survey: 2005 emergency department summary. Advance Data 2007;386:1-32.

5 Goodacre S, Thokala P, Carroll C, et al. Systematic review, metaanalysis and economic modelling of diagnostic strategies for suspected acute coronary syndrome. Health Technol Assess 2013;17:v-vi, 1-188.

6 Gorenberg M, Marmor A, Rotstein H. Detection of chest pain of non-cardiac origin at the emergency room by a new non-invasive device avoiding unnecessary admission to hospital. Emerg Med J 2005;22:486-9.

7 Knockaert DC, Buntinx F, Stoens N, et al. Chest pain in the emergency department: the broad spectrum of causes. Eur J Emerg Med 2002;9:25-30.

$8 \mathrm{Mol} \mathrm{KA}$, Rahel BM, Meeder JG, et al. Delays in the treatment of patients with acute coronary syndrome: focus on pre-hospital delays and non-ST-elevated myocardial infarction. Int $J$ Cardiol 2016;221:1061-6.

9 Backus BE, Six AJ, Kelder JC, et al. Chest pain in the emergency room: a multicenter validation of the heart score. Crit Pathw Cardiol 2010;9:164-9.

10 Stopyra JP, Harper WS, Higgins TJ, et al. Prehospital modified heart score predictive of 30-day adverse cardiac events. Prehosp Disaster Med 2018;33:58-62.

11 Alghamdi A, Howard L, Reynard C, et al. Enhanced triage for patients with suspected cardiac chest pain: the history and Electrocardiogram-only Manchester acute coronary syndromes decision aid. Eur J Emerg Med 2019;26:356-61.

12 Aarts GWA, Camaro C, van Geuns R-J, et al. Acute rule-out of nonST-segment elevation acute coronary syndrome in the (pre)hospital setting by HEART score assessment and a single point-of-care troponin: rationale and design of the ARTICA randomised trial. BMJ Open 2020;10:e034403.

13 Ambulancezorg Nederland. Landelijk protocol Ambulancezorg 8.1, 2016. Available: https://www.ambulancezorg.nl/themas/kwaliteitvan-zorg/protocollen-en-richtlijnen/landelijk-protocol-ambulancezorg

14 Backus BE, Six AJ, Kelder JC, et al. A prospective validation of the heart score for chest pain patients at the emergency department. Int $J$ Cardiol 2013;168:2153-8.

15 Philips. Tempus pro monitor (Philips), 2019. Available: https://www. rdtltd.com/

16 Philips. IntelliSpace Corsium (Philips), 2019. Available: https://www. philips.co.uk/healthcare/product/HC881072/intellispace-portal-90advanced-visual-analysis

17 FDA approval IntelliSpace Corsium (Philips). Fda primary device ID: 05060472441331, 2019. Available: https://fda.report/GUDID/ 05060472441331

18 Liem S-S, van der Hoeven BL, Oemrawsingh PV, et al. Mission!: optimization of acute and chronic care for patients with acute myocardial infarction. Am Heart J 2007;153:14.e1-14.e11.

19 Ishak M, Ali D, Fokkert MJ, et al. Fast assessment and management of chest pain without ST-elevation in the pre-hospital gateway: rationale and design. Eur Heart $J$ 2015;4:129-36.

20 Ishak M, Ali D, Fokkert MJ. Fast assessment and management of chest pain patients without ST-elevation in the pre-hospital gateway (famous triage): ruling out a myocardial infarction at home with the modified heart score. European Heart Journal: Acute Cardiovascular Care 2018;7:102-10.

21 van Dongen DN, Tolsma RT, Fokkert MJ, et al. Pre-Hospital risk assessment in suspected non-ST-elevation acute coronary syndrome: a prospective observational study. Eur Heart $J$ 2020;9:5-12. 
22 van Dongen DN, Tolsma RT, Fokkert MJ, et al. Referral decisions based on a prehospital heart score in suspected non-ST-elevation acute coronary syndrome: design of the famous triage 3 study. Future Cardiol 2020;16:217-26. 